\title{
Kicked drops
}

\author{
Pierre Chantelot, ${ }^{1,2}$ Martin Coux, ${ }^{1,2}$ Lucie Domino, ${ }^{1}$ Benoît Pype,,${ }^{1,3}$ Christophe Clanet,${ }^{1,2}$ \\ Antonin Eddi, ${ }^{1}$ and David Quéré ${ }^{1,2}$ \\ ${ }^{1}$ Physique et Mécanique des Milieux Hétérogènes, UMR 7636 du CNRS, ESPCI, 75005 Paris, France \\ ${ }^{2}$ LadHyX, UMR 7646 du CNRS, École polytechnique, 91128 Palaiseau, France \\ ${ }^{3}$ PSL-SACRe, EA 7410, EnsadLab, 75005 Paris, France
}

(Received 2 February 2018; published 17 October 2018)

\begin{abstract}
This paper is associated with a video winner of a 2017 APS/DFD Milton van Dyke Award for work presented at the DFD Gallery of Fluid Motion. The original video is available from the Gallery of Fluid Motion, https://doi.org/10.1103/APS.DFD.2017.GFM.V0080
\end{abstract}

DOI: 10.1103/PhysRevFluids.3.100503

Vibrating their substrate is an efficient way to manipulate drops and even to sculpt them, as recently discussed in the literature: (1) drops on a shaken inclined plate can surprisingly climb the slope [1]; (2) a puddle on a horizontal vibrating material deforms with asymmetric shapes [2]; (3) an impulsive motion of the substrate generates superpropulsion, that is, an enhanced takeoff of the deposited liquid [3]. Here we report the striking shapes obtained when subjecting water pearls to a strong, quick, vertical impulse.

Figure 1 shows the evolution of a water puddle (volume of about $200 \mu \mathrm{l}$ ) accelerated by $\sim 100 \mathrm{~g}$ ( $g$ being the acceleration of gravity), with a corresponding upward displacement of $\sim 10 \mathrm{~mm}$. In this experiment, we first deposit dyed water on a superhydrophobic substrate itself placed on a shaker. We use the two rows of Fig. 1 to decompose the main steps of the drop behavior: (1) as the plate moves up, the globular puddle first spreads out to form a disk whose edges take off as the substrate decelerates; (2) water then adopts the shape of a conical cavity whose base contacts the substrate. This cavity is subjected to surface tension and inertia, which makes its shape evolve and eventually collapse, leading to the eruption of a jet and to the formation of numerous satellite droplets.

These liquid vases can be sculpted by playing on the substrate nature and on the number of drops initially placed on the device. Contrasting with our usual way of looking at the simplest possible device to make generic behaviors emerge, we considered here complex substrates, so as to generate unique water shapes. To that end, we use a pedestal heightened by a thin fraction of solid tube where water is placed. The choice of a tube allows us to mix effects of solid curvature to that generated by the nonaxisymmetry of the device: the liquid is guided by the tube walls but it can leak from the tube ends. The different pieces are eventually attached to the shaker, glued together and made superhydrophobic, and we show in Fig. 2 three examples of the ephemeral water sculptures arising from these complex substrates. From left to right, we increase the number of deposited drops from one [Fig. 2(a)] to two [Fig. 2(b)] and then four [Fig. 2(c)]; multiple drops are separated by a superhydrophobic wire, which can be seen in photographs (b) and (c). We impose an upward impulsive motion (acceleration comparable to that in Fig. 1) and capture the transient shapes adopted by water in both the spreading stage (top row) and in the takeoff stage (bottom row). These pictures highlight the extreme variety

Published by the American Physical Society under the terms of the Creative Commons Attribution 4.0 International license. Further distribution of this work must maintain attribution to the author(s) and the published article's title, journal citation, and DOI. 


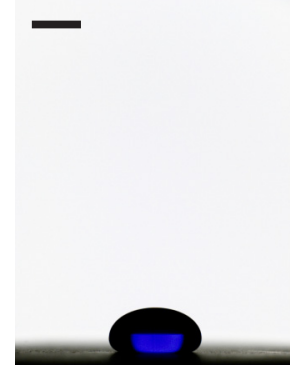

$t=0 \mathrm{~ms}$

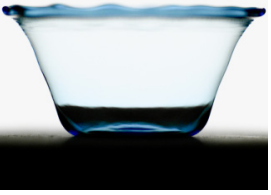

$t=16 \mathrm{~ms}$

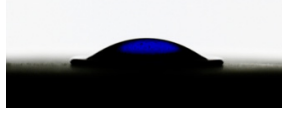

$t=4 \mathrm{~ms}$

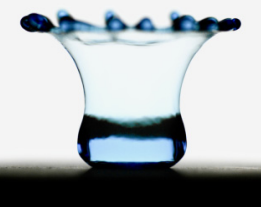

$t=22 \mathrm{~ms}$

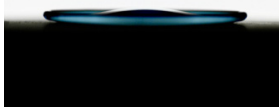

$t=7 \mathrm{~ms}$

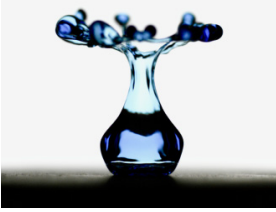

$t=26 \mathrm{~ms}$

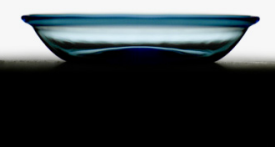

$t=10 \mathrm{~ms}$

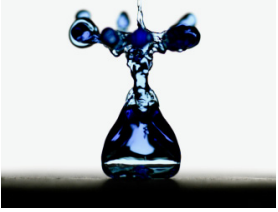

$t=28 \mathrm{~ms}$

FIG. 1. Chronophotography of a small water puddle deposited on a superhydrophobic plate subjected to an impulse. As the plate is accelerated upward, water spreads in a disk whose edges take off. Then, the liquid drop forms a truncated cone that eventually collapses, leading to the creation of a jet and satellite droplets. The scale bar is $5 \mathrm{~mm}$. The video associated with these images is available at https://doi.org/10.1103/APS.DFD.2017.GFM.V0080
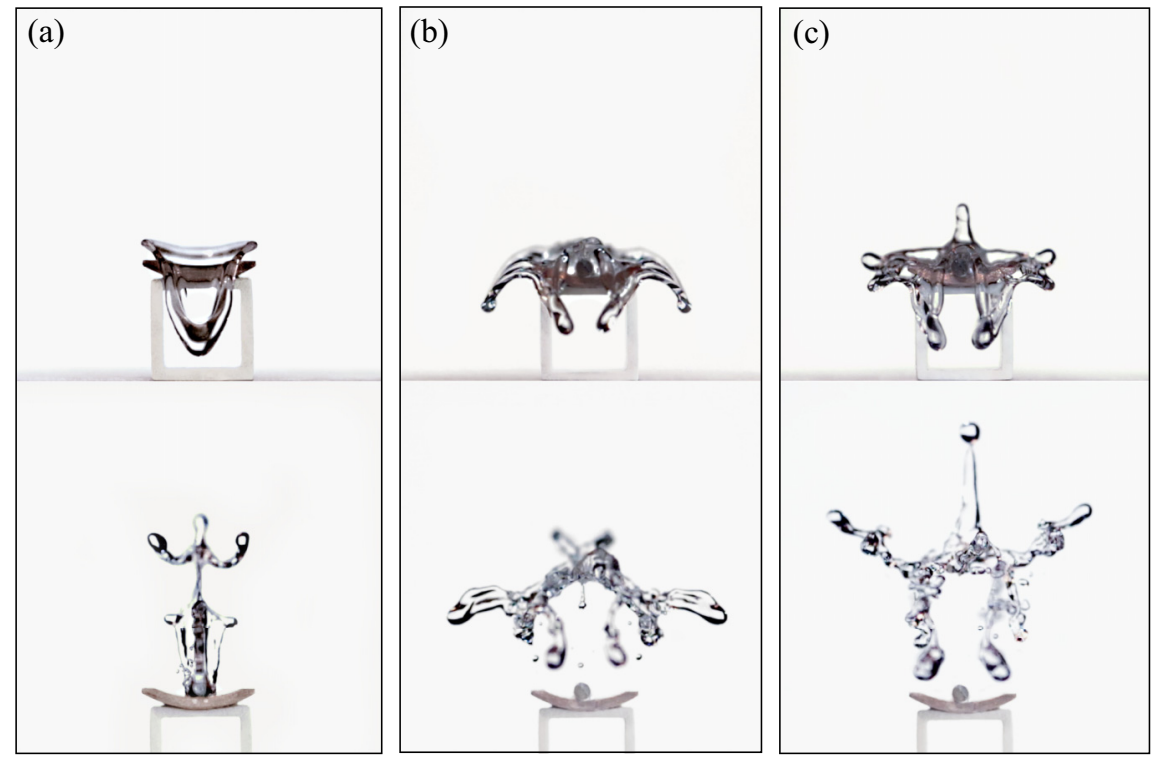

FIG. 2. Transient shapes captured during the spreading (top row) and takeoff (bottom row) of water drops deposited on superhydrophobic curved pedestals. Increasingly complex shapes are created by depositing one drop (a), two drops separated by a repellent wire (b), and four drops, two on each side of the wire (c). 
of shapes we can design, some of them being even vaguely anthropomorphic. In the physics of droplet impact, the interplay between moving liquids and their substrate leads to elegant structures via complex dynamics. Here we amplify these phenomena by using specifically designed substrates and a water-repellent coating to obtain richer and more intricate shapes.

We thank Direction Générale de l'Armement (DGA) for financial support.

[1] P. Brunet, J. Eggers, and R. D. Deegan, Vibration-Induced Climbing of Drops, Phys. Rev. Lett. 99, 144501 (2007).

[2] X. Noblin, A. Buguin, and F. Brochard-Wyart, Triplon Modes of Puddles, Phys. Rev. Lett. 94, 166102 (2005).

[3] C. Raufaste, G. R. Chagas, T. Darmanin, C. Claudet, F. Guittard, and F. Celestini, Superpropulsion of Droplets and Soft Elastic Solids, Phys. Rev. Lett. 119, 108001 (2017). 